\title{
A Flight Experiment of Electrodynamic Tether Using HTV toward the Realization of Debris Removal
}

\author{
By Satomi Kawamoto, ${ }^{1)}$ Yasushi OhKawa, ${ }^{1)}$ Kentaro IKI, ${ }^{1)}$ Teppei OKUmURA, ${ }^{1)}$ JunIchi AoYama, ${ }^{1)}$ \\ Yasuhiro KAtAYAmA, ${ }^{1)}$ Daisuke TsujitA, ${ }^{2)}$ Toru KAsAI, ${ }^{3)}$ Hirohiko UEMATSU ${ }^{3)}$ and Koichi InouE ${ }^{4}$ \\ ${ }^{1)}$ Research and Development Directorate, JAXA, Chofu, Japan \\ ${ }^{2)}$ Mitsubishi Heavy Industry, Komaki, Japan \\ ${ }^{3)}$ Human Spaceflight Technology Directorate, JAXA, Tsukuba, Japan \\ ${ }^{4)}$ Research and Development Directorate, JAXA, Tsukuba, Japan
}

(Received August 8th, 2015)

\begin{abstract}
The active removal of existing space debris is the one of the most proactive strategies to suppress space debris growth. The Japan Aerospace Exploration Agency (JAXA) is investigating a cost-effective space debris removal system that employs the highly efficient electrodynamic tether (EDT) technology as its orbital transfer system. As the first step toward the realization of a debris removal system, a flight experiment of EDT using the H-II Transfer Vehicle (HTV, or "Kounotori") is planned. This experiment is called the Kounotori Integrated Tether Experiment (KITE). The purpose of the KITE mission is to establish and demonstrate EDT technology and to obtain some EDT characteristics, such as flexible tether dynamics, and electron emission and its collection in space plasma. Precise numerical simulations were performed to analyze some aspects of mission by modeling the tether as a lumped mass. This method can account for its flexibility by segmenting the tether into point masses connected by segments comprising a spring and viscous damper. In the KITE mission, some interesting operations such as tether libration suppression using thrusters on HTV are planned. This tether control uses a thruster, which enables various operations such as collision avoidance maneuvers, that is required for debris removal satellites in the future. In this paper, the results of the numerical simulations of the KITE mission and their potential applications are presented.
\end{abstract}

Key Words: Active Debris Removal, Electrodynamic Tether, H-II Transfer Vehicle

\section{Introduction}

The quantity of debris increases with the number of collisions, explosions, and other factors. The increase in the number of debris collision avoidance maneuvers (CAMs) and the actual occurrence of collisions is becoming a serious problem for continued developments in space and the associated applications for humankind. Various types of debris ranging from intact large objects to small fragments debris in circulation already exist. Debris that is large enough to be tracked from the ground can be avoided by CAM if a spacecraft has thrusters, and some critical parts of a spacecraft can be protected from hypervelocity impact of tiny debris fragments by shielding. These countermeasures against debris are indispensable today; they not only offer protection to the spacecraft but also maintain an optimally clear environment in space. However, debris ranging from some millimeters to centimeters cannot be tracked nor protected at this moment. Although we are making an effort to track the smaller debris and protect from the larger debris, but it seems to be difficult to fill a gap soon, and they continue to pose a risk to spacecraft. While these risks are still considered to be acceptable at present, the future utility of space can only be guaranteed with the introduction of countermeasures to effectively reduce the increasing risks, costs and burdens imposed by the presence of debris. Moreover, the mutual collisions between debris are predicted and the quantity of debris will continue to increase even with the good compliance of commonly adopted mitigation measures. ${ }^{1)}{ }^{2)}$ Remediation measures, such as active debris removal (ADR), should be considered to stabilize the future LEO environment. Direct risks are posed by debris fragments; but it is inefficient to remove only small debris objects that have already spread over a significant amount of space because significant number of fragments are generated by collisions between large debris objects. It has been estimated that by removing 5 to 10 large, intact debris objects in crowded orbits per year, the overall number of catalogued debris objects can be maintained at the present level in the future. However, if the cost required for ADR is too expensive, alternative debris countermeasures, such as launching replacement spacecraft in case a spacecraft is lost due to debris collision are still less expensive and ADR will not be realized. However, the cost of launching replacement spacecraft is increasing annually. If the cost of debris countermeasures without ADR increases in the future, it will be too late or too expensive to start using ADR after a sufficient number of fragments have already spread over space. The cost of debris countermeasures including ADR will be the total space development cost. Thus, for continued development in space for the utilization of associated applications by humankind, the cost of ADR should be reasonable. Electrodynamic tether (EDT) technology has been identified as one of the potentially cost effective measures to remove debris. It is characterized by simple and highly efficient features and its ease for attaching to debris. Previous studies showed that EDT can generate sufficient thrust for debris deorbiting and it is promising for the cost effective ADR. ${ }^{3)}$ However, on-orbit dynamics of a flexible and conductive tether and the interaction between the ambient plasma is less well- 
established. The Japan Aerospace Exploration Agency (JAXA) is planning a flight demonstration to establish EDT technologies using the HTV6 toward the realization of ADR. This paper investigates the dynamics that characterize this mission and the applications for a debris removal satellite (DRS) in the future.

\section{Electrodynamic Tether System for Debris De-oribiting}

The principle of an EDT thrust is described in this section. When a tether is deployed from a spacecraft in orbit, it is vertically stabilized by the gravity gradient force. An electromotive force is set up within a conductive tether as it moves through the geomagnetic field in its orbit around the Earth. If a cathode at the end of the tether where electric potential is low emits electrons, the electrons are collected from the ambient plasma by the other end of the tether (i.e., on the side with higher electric potential), if the tether is bare (i.e., uninsulated). ${ }^{4)}$ The electric current flows through the tether by closing the circuit via the ambient plasma. The tether then generates a Lorentz force due to the interaction between the current and the geomagnetic field (Fig. 1). Therefore, EDT systems can provide deceleration without the need for a propellant. Previous studies showed that debris objects that should be removed from crowded orbits can reenter the Earth's atmosphere within one year with a 10km EDT. ${ }^{3)}$ The Lorentz force is small enough and no thrust vector control is required throughout the de-orbiting phase. Hence, the tether can be attached anywhere onto a debris object. Thus, the operation is comparatively less challenging than fixing a conventional thruster to the target, which requires it to be fixed to strong points and in the right direction. Some simple methods for attaching the tether to debris that does not require precise position and attitude control have been studied. These include the use of extensible booms or harpoons/punchers. ${ }^{5)-8)}$

EDT does not require a significant amount of power or large amounts of fuel. Hence, it is expected to realize ADR using a small satellite or to facilitate multi removal by using a single removal satellite with EDT packages to enable ADR at a low cost. At present, solutions with EDT have not yet yielded significant results. A demonstration in orbit was planned to

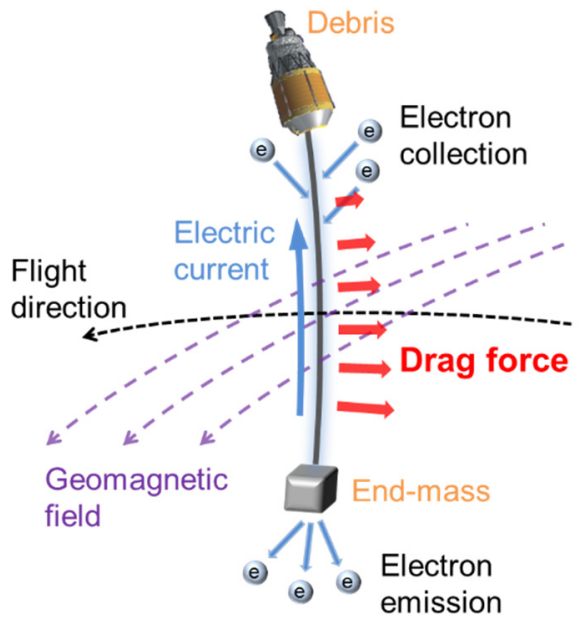

Fig. 1. The principle of EDT. address these knowledge gaps and to better understand some characteristics of interest such as tether deployment and electron emission and collection.

\section{EDT Demonstration using HTV}

\subsection{Mission concepts and objectives}

This section describes the mission that was planned to demonstrate the utility of HTV. After the HTV delivers cargo/supplies to the International Space Station (ISS) and departs, it lowers its altitude by more than $20 \mathrm{~km}$ from the ISS' altitude. An end-mass is ejected by a spring mechanism installed on the zenith side of the HTV (Fig. 2). A spool of an approximately $700 \mathrm{~m}$ tether is installed in the end-mass; the other end of the tether is connected to the HTV. The tether is released from the inside of the tether spool and the deployment friction will gradually decrease the velocity of the end-mass. However, after the tether has been deployed for a significantly long time, the gravity gradient force will accelerate the tether deployment. A spool type reel deployed from the inside was chosen because the amount of friction is less than a spool type reel deployed from the outside or with the use of a dram type reel. If the deployment friction is larger than expected, the tether deployment stops. A brake reel is installed on the endmass to stop the deployment smoothly, to avoid the buildup to a tension peak that would otherwise severe the tether. The tether will exhibit notable libration due to the Coriolis force generated during the deployment phase, the torque generated by the Lorentz force or the air drag over the tether. The deployment friction or libration damping of the tether cannot be evaluated on the ground; hence, an in-orbit demonstration is required. These deployment dynamics are measured by a rendezvous radar onboard the HTV that is used to rendezvous to the ISS. Only reflectors on the end-mass are needed for the measurement of its relative position and it does not require any electronic devices. Cameras are also installed on the HTV for monitoring the tether deployment in the initial phase and the tether librations afterward. The field emission cathode (FEC) onboard the HTV emits electrons that initiate the flow of

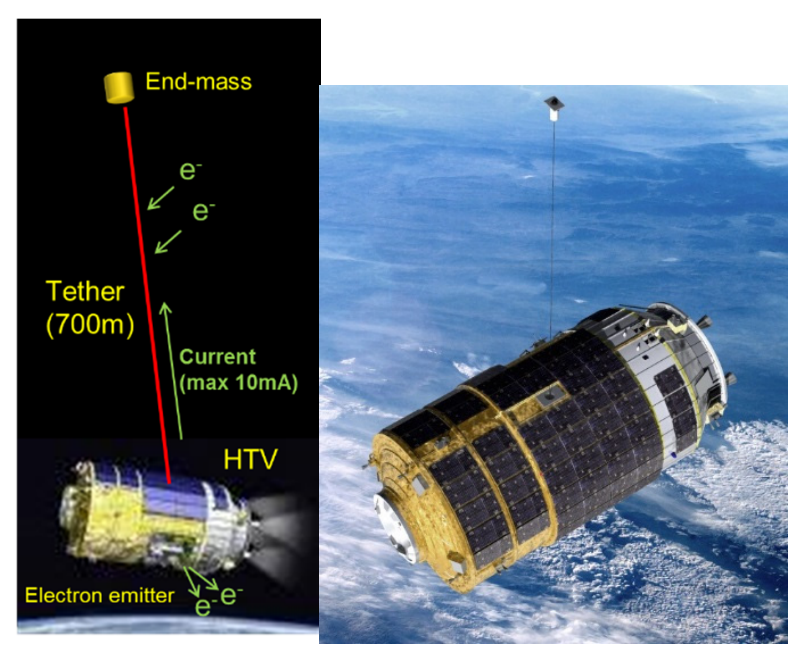

Fig. 2. The experiments configuration. 


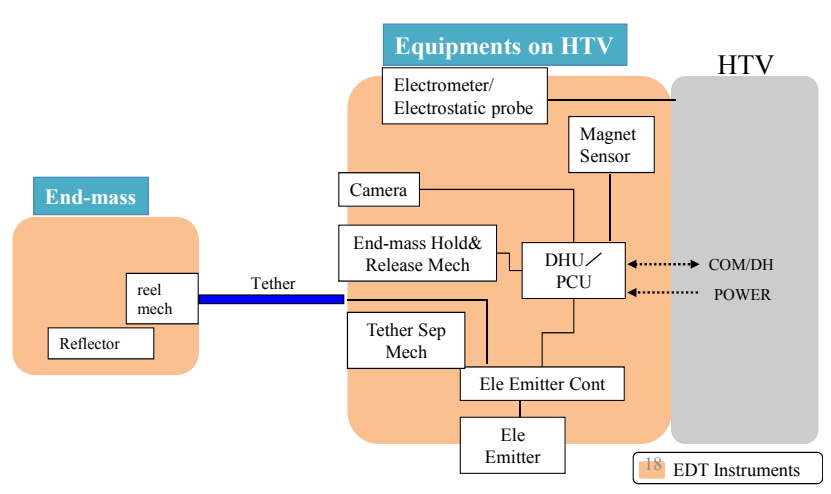

Fig. 3. System block diagram.

electric current through the tether. The electric potential, with respect to the space plasma, is measured by a plasma potential monitor and the geomagnetic field is measured by a geomagnetic sensor. These components are operated using sources of power and by means of communication provided by the HTV. Fig. 2 and Fig. 3 illustrate the configuration and system block, respectively. After the tether has been deployed, the relations between the electric potential and current and an autonomous FEC control is examined. The mission is planned for 7 days. After the mission has been completed, the tether will be cut and the HTV is scheduled for reentry. Further details about the KITE mission are described in 9) and 10).

\subsection{Roadmap toward debris removal}

Our roadmap for developing a debris removal system with an EDT is shown in Fig. 4. The final goal is to develop a cost- effective debris removal system with international cooperation to preserve the space environment. Although the debris removal system requires a range of advanced technologies (e.g., autonomous rendezvous and the capture of non-cooperative, uncontrolled objects), the development of a highly efficient propulsion system is the most fundamental and critical requirement because requirements of capturing depend on the propulsion system for de-orbiting. If EDT can be used for debris de-orbiting, the process of attaching the tether to the debris object is less challenging, compared with that with the conventional propulsion system. Furthermore, a small satellite can be used for the DRS since a large amount of fuel is not required for EDT. Therefore, the first step toward realizing the debris removal system is to establish EDT technology. The next step of our roadmap is to develop technologies for removing one large debris object with a small satellite. If EDT technologies can be confirmed by the first step and the desired characteristics are obtained, we will be able to use these results to design a system demonstration of ADR. For removing large debris objects with the mass of about 1 to 3 tons in the crowded regions (altitude of $800-1000 \mathrm{~km}$ ), EDT will require a tether length of 5-10 km and several Ampères of current. Noncooperative rendezvous and proximity operations such as motion estimation and the attachment of the tether end to debris object are now being studied in parallel. An international framework is also needed for realizing a practical ADR in the future. ${ }^{11)}$

In the system demonstration, we plan to use the upper stage of a rocket that launches the small satellite as the target to be

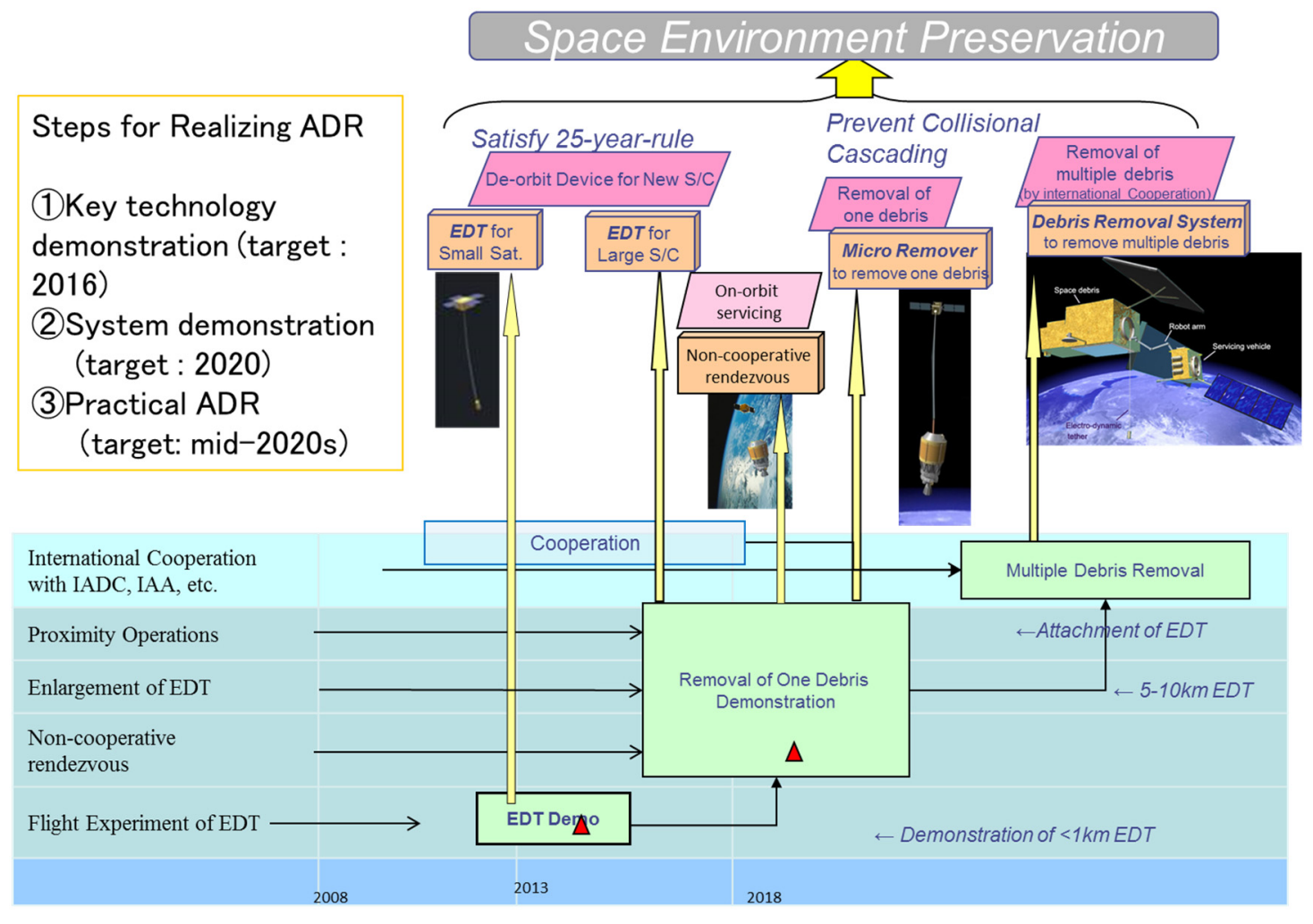

Fig. 4. Roadmap of debris removal with EDT. 
removed. This small satellite will be launched alongside new satellites, rendezvous with the upper stage again after its separation, and attach the end of the tether to the upper stage for de-orbiting. The small satellite becomes the end-mass of the tether and de-orbits along with the upper stage. This use of a small satellite is one way for debris removal of practical use in the final goal, other than using dedicated DRSs for multi debris removal, since the launch cost can be greatly reduced for the small satellite.

\section{Numerical Simulations of EDT}

Since the tether is more than several hundreds of meters in length that operates in a vacuum or plasma non-gravitational environment, it cannot be evaluated on ground. Therefore, these in-orbit dynamics are studied using numerical simulations. ${ }^{12)}$

\subsection{Modeling and the equation of motion}

The tether is modeled as point masses connected by springs and viscous dampers. The motion equation of each point mass is formulated in the coordinate system whose origin is at the center of mass of the system rotating around the Earth (Fig. 5). The equation of the motion of the $i$-th point mass in this rotating coordinate is as follows:

$$
\begin{aligned}
\ddot{x}_{i}= & \left(r+y_{i}\right) \ddot{\theta}+2\left(\dot{r}+\dot{y}_{i}\right) \dot{\theta}+x_{i} \dot{\theta}^{2} \\
& -\mu_{e} x_{i}\left[x_{i}^{2}+\left(y_{i}+r\right)^{2}+z_{i}^{2}\right]^{-\frac{3}{2}}+\frac{Q_{x i}}{m_{i}} \\
\ddot{y}_{i}= & -\ddot{r}-x_{i} \ddot{\theta}-2 \dot{x}_{i} \dot{\theta}+\left(r+y_{i}\right) \dot{\theta}^{2} \\
& -\mu_{e}\left(y_{i}+r\right)\left[x_{i}^{2}+\left(y_{i}+r\right)^{2}+z_{i}^{2}\right]^{-\frac{3}{2}}+\frac{Q_{y i}}{m_{i}} \\
\ddot{z} & =-\mu_{e} z_{i}\left[x_{i}^{2}+\left(y_{i}+r\right)^{2}+z_{i}^{2}\right]^{-\frac{3}{2}}+\frac{Q_{z i}}{m_{i}}
\end{aligned}
$$

where $\dot{\theta}, r, \mu_{e}$ represent the orbital angular velocity of the C.G. of the tether system, the radius of the orbit, and the gravitational constant of the Earth, respectively. $m$ is the mass of the point, and $Q$ is the total force acting on it. Orbital perturbations caused by the Lorentz force, air drag, and geo-potential are considered using Gauss's variational equations of motion. The deployment of the tether is modeled by adding new point masses. Parameters such as tensile modulus, damping ratio, deployment friction, and optical thermal characteristics of the tether are measured by tether-based experiments, described in 13).

\subsection{Other models}

The following models are used, IGRF 2000 (International Geomagnetic Reference Field) $(10 * 10)$ for the geomagnetic field, IRI2001 (International Reference Ionosphere) for plasma density, NRLMSISE-00 (NRL Mass Spectrometer, Incoherent

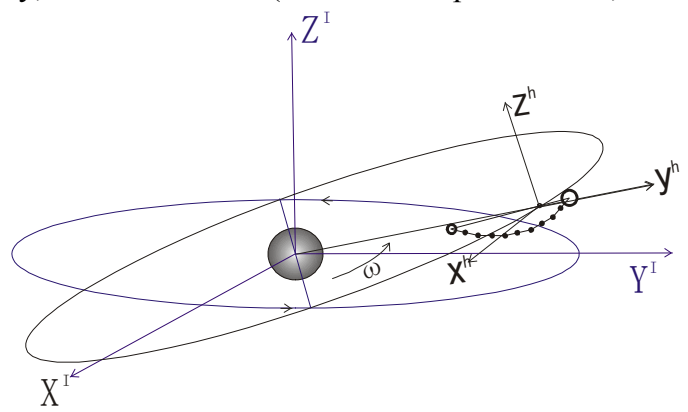

Fig. 5. Rotating coordinate around the Earth $\left(\mathrm{X}^{\mathrm{h}}, \mathrm{Y}^{\mathrm{h}}, \mathrm{Z}^{\mathrm{h}}\right)$.
Scatter Radar Extended Model) for atmospheric density, and EGM96 (Earth Gravitational Model) $(10 * 10)$ for the Earth's geo-potential field. For electron collection by the bare tether, the two-dimensional orbital motion limit (OML) theory described below was used.

$$
\frac{d J}{d x}=e n_{p} d\left(\frac{2 e \phi(x)}{m_{e}}\right)^{\frac{1}{2}}
$$

where $J(x), \Phi(x) e, n_{p}, d$, and $m_{e}$ represent the current, potential, the elementary electron charge, the plasma density, tether diameter, and the electron mass, respectively.

The temperature of the tether is calculated and the natural length and the conductivity of the tether vary as a function of the temperature.

\subsection{Parameters used in the simulations}

Parameters of the tether system for the KITE mission are shown in Table 1.

Table 1. Parameters of numerical simulations for the KITE mission.

\begin{tabular}{|l|l|}
\hline Tether mass & $0.7 \mathrm{~kg}$ \\
\hline End-mass & $19.3 \mathrm{~kg}$ \\
\hline HTV & $13000 \mathrm{~kg}$ \\
\hline Tether length & $700 \mathrm{~m}$ \\
\hline Tether diameter & $0.001 \mathrm{~m}$ \\
\hline orbit & $400 \mathrm{~km}$ alt. $52^{\circ}$ inclination \\
\hline
\end{tabular}

\section{Numerical Simulation Results}

\subsection{Libration}

Fig. 6 shows the dynamics of the tether during deployment. The in-plane libration angle during and after the deployment phase is shown in Fig. 7. The end-mass is ejected by $1 \mathrm{~m} / \mathrm{s}$ in the zenith direction, inclined at an angle of $20^{\circ}$ in the orbital velocity direction and $11.25^{\circ}$ to the out-of-plane. It is intentionally ejected at $20^{\circ}$ within the orbital plane in order to keep the end-mass inside the field of view of the RVS as long as possible. The angle $11.25^{\circ}$ to the out-of-plane is caused by the restriction of installation on the HTV. Large in-plane libration is predicted to occur due to the Coriolis force. The details of the deployment dynamics are shown in 14).

\subsection{Pitch angle control of HTV}

Since the field of view of the rendezvous radar on the HTV is $\pm 20^{\circ}$ in both the azimuth and the elevation, the end-mass is out of view when the tether exhibits libration amplitudes $>20^{\circ}$. To keep measuring the position of the end-mass, the HTV will control its pitch angle to track the end-mass in its field of view. Fig. 8 shows the pitch angle and the angular velocity of the HTV when the HTV controls its pitch angle for $30^{\circ}$ with the angular velocity of $0.5^{\circ}$ per second and Fig. 9 shows the inplane libration angle of the tether with and without this operation. The tether is long enough and is released from the spool reel on the end-mass. Consequently, the motion of the other side of the tether connected to the HTV does not affect the dynamics of its deployment. 


\subsection{Libration suppression by HTV thrust}

After the libration motion of the tether is measured for some period, HTV thrusters are used to suppress the tether libration. If the thrust is initiated with proper timing (e.g., when the endmass with respect to the HTV moves in the opposite direction of the orbital velocity, the thrust should be in the same direction with the end-mass, that is, the opposite direction of the orbital velocity) and the libration angle can be decreased (Fig. 10). In Fig. 11, two thrusts at $\mathrm{dV}=0.2 \mathrm{~m} / \mathrm{s}$ are given to decrease the tether libration angle. The altitude of the orbit can be maintained constant by lowering and raising the altitude (Fig. 12).

\subsection{Electric potential}

The EMF is measured using an electrometer after the tether is deployed. Fig. 13 shows the expected change in the EMF when $700 \mathrm{~m}$ of tether is deployed. If the FEC is not working, the tether potential, with respect to the space plasma, depends on the balance between electron collection by end-mass side of the tether and ion collection by the HTV side of the tether. The mass of ions is greater than that of electrons; thus, the potential of the majority of the tether will be lower than the plasma potential. When electrons are emitted from the FEC, they are collected from the ambient plasma by the end-mass side tether, where the electric potential with respect to the space plasma becomes higher and electric currents flow through the tether. Fig. 14 shows the electric potentials of the end-mass and the HTV with respect to the space plasma, when a maximum 10 $\mathrm{mA}$ current flows through the tether.

The Lorentz force is generated when the electric current flows through the tether, but it is too small to change the orbit of the heavy HTV. It is around one-hundredth smaller than the air drag force acting on HTV as its altitude is low (400 km).

\subsection{Libration generation by Lorentz force}

Since the masses of the HTV and end-mass are quite different, the center of mass is not located in the middle of the tether. When the Lorentz force acts on the tether, it causes the tether to be inclined and libration growth can be observed because the torque generated by the Lorentz force causes the tether to turn away from the local vertical. If the electric current is switched on and off in time to the tether libration angle, the libration amplitude grows efficiently. Fig. 15 demonstrates the out-ofplane libration growth by controlling the electric current in a recurrent on and off state compared with how the tether continues to liberate with $5^{\circ}$ of amplitude in the case when a continuous electric current is applied. It is important to suppress the initial libration amplitude so that it is small enough to confirm the libration growth by the Lorentz force. Therefore, libration suppression by HTV thrust is required before starting this operation.

\subsection{The effect of HTV thrust}

If a strong thrust is applied to the HTV, the tether can start tumbling. For example, if $\mathrm{dV}=2.0 \mathrm{~m} / \mathrm{s}$ is applied to the HTV, the tether starts to rotate unless the thrust is applied with proper timing (Fig. 16). Thus, if the HTV needs to activate debris collision avoidance maneuvers, the quantity and the timing of the $\mathrm{dV}$ is limited. When larger orbit changes are required for HTV's controlled reentry, the tether will start tumbling independent of the timing.

\subsection{Tether cut}

A rotating tether can be an obstacle for the safe reentry of the HTV, the tether should be cut after the KITE mission before the HTV reenters. When the tether is cut, the end-mass and the tether will be in a higher orbit. The end-mass with the tether will have an apogee altitude that is $5 \mathrm{~km}$ higher than the original altitude when the tether is cut under stable, vertical conditions (Fig. 17), and doubles when the tether is liberating (Fig. 18). This is the reason why the KITE mission should be conducted more than $20 \mathrm{~km}$ below the ISS orbit, in case for an unexpected tether cut.

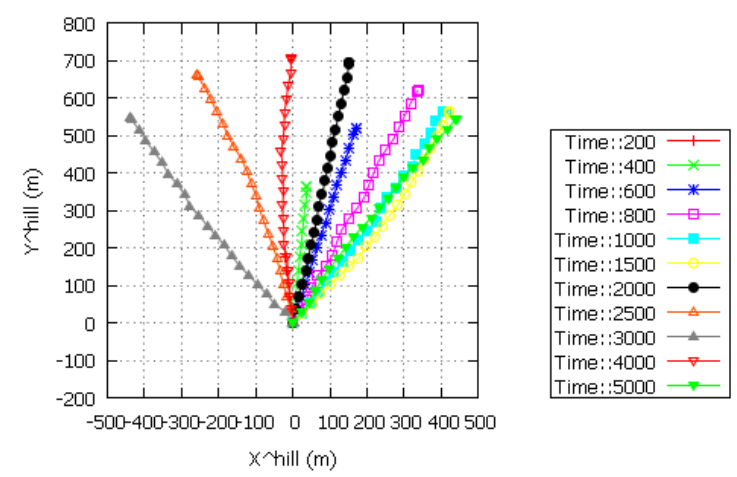

Fig. 6. Change in the form of the tether during the deployment phase.

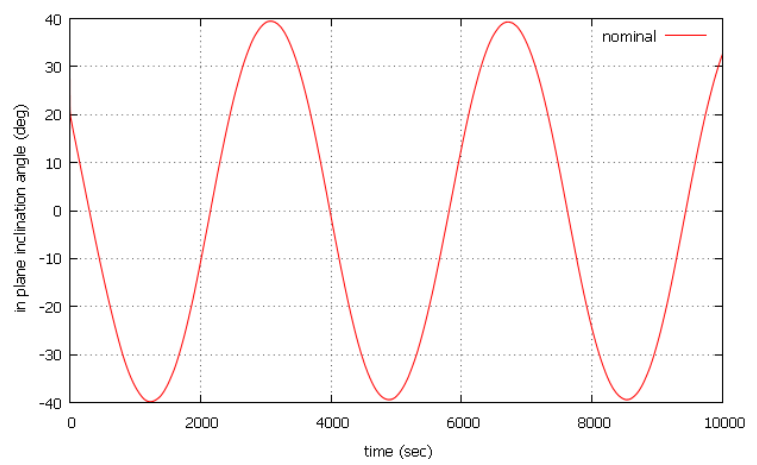

Fig. 7. Change in the in-plane libration angle during and after deployment.

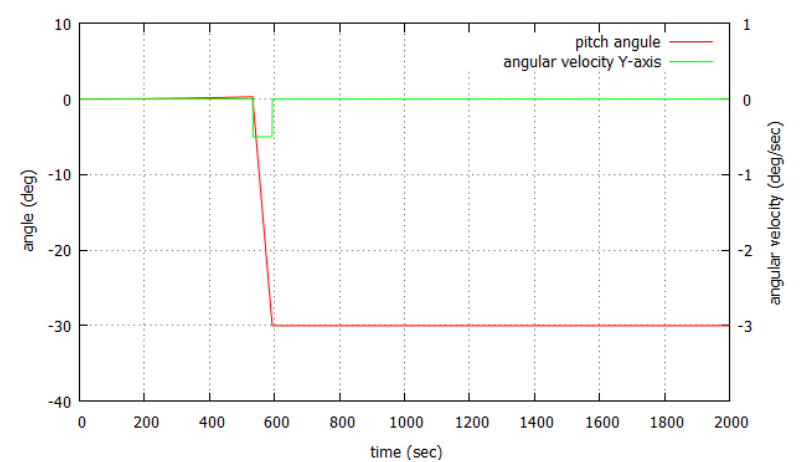

Fig. 8. Change in the pitch angle and the angular velocity of the HTV when its pitch angle changes at $30^{\circ}$ to track the end-mass inside the field of view of the RVS. 


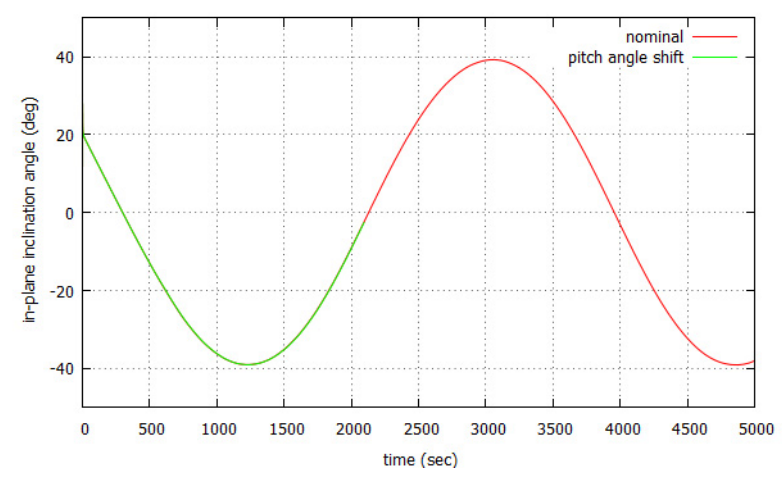

Fig. 9. Change in the in-plane libration angle with and without pitch maneuvers.

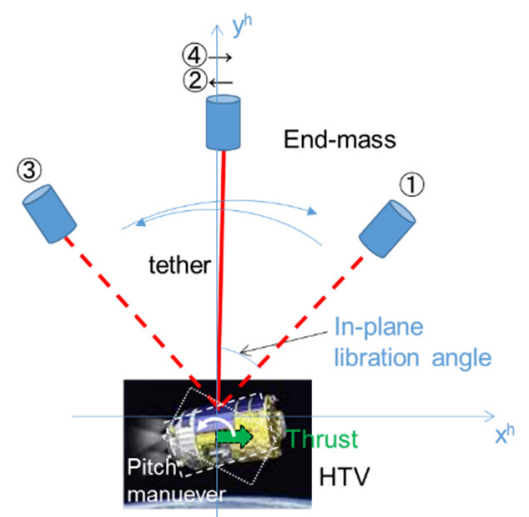

Fig. 10. The timing to apply thrust for libration suppression.

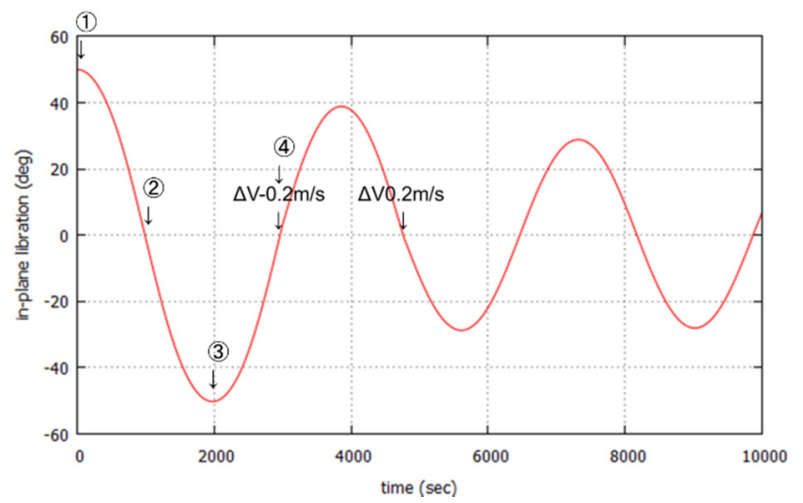

Fig. 11. Change in in-plane libration angle with two thrusts for libration suppression.

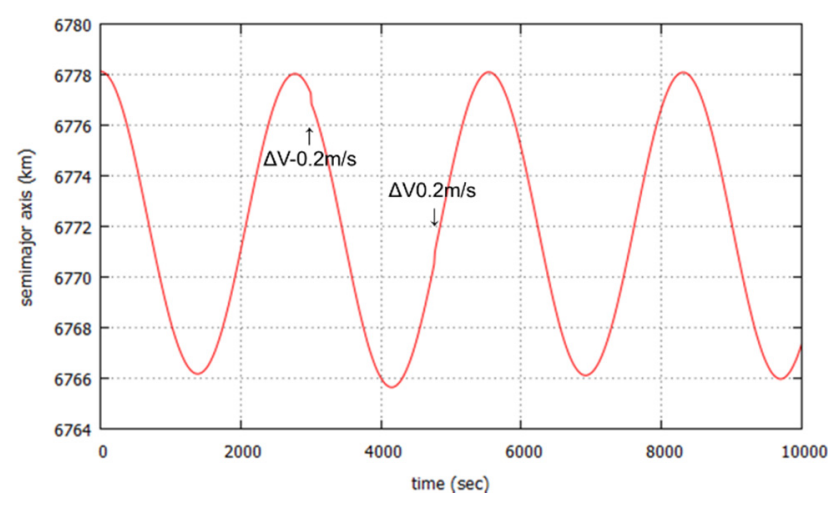

Fig. 12. Change in the altitude with libration suppression thrusts.

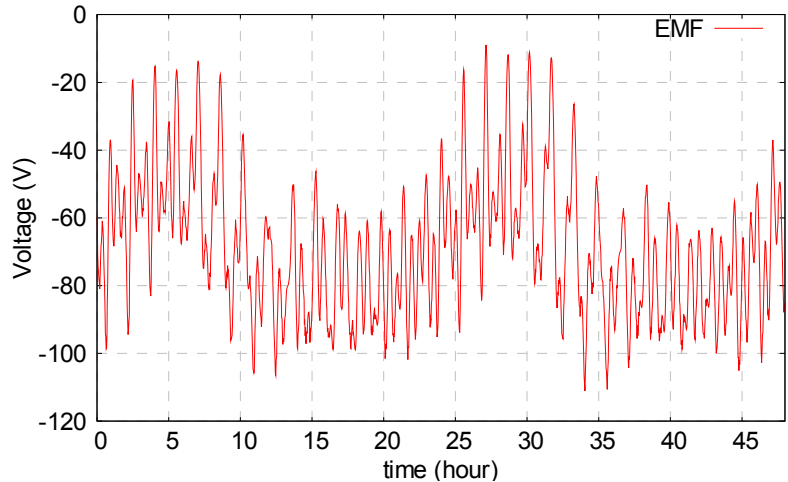

Fig. 13. Expected EMF when $700 \mathrm{~m}$ tether is deployed.

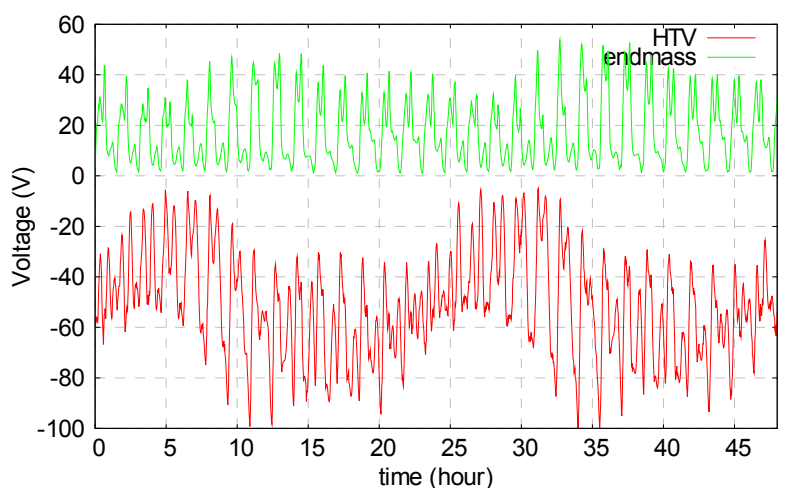

Fig. 14. Electric potential with respect to space plasma.

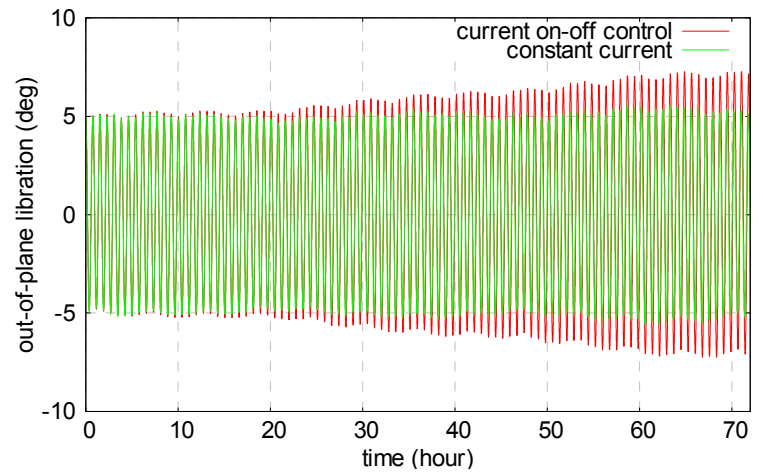

Fig. 15. Tether libration growth by switching the electric current.

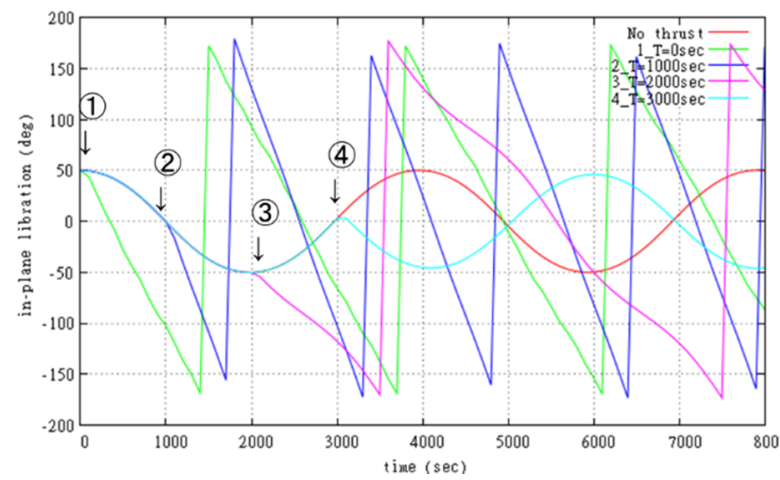

Fig. 16. Tether libration when $\mathrm{dv}=2.0 \mathrm{~m} / \mathrm{s}$ is applied. The tether will rotate when the $\mathrm{dV}$ is applied, except timing (4). 


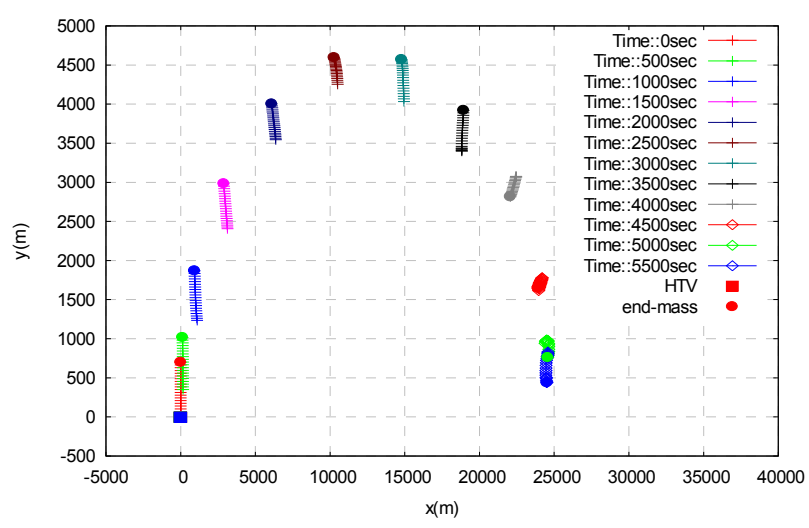

Fig. 17. Tether behavior when the tether is cut under stable, vertical conditions.

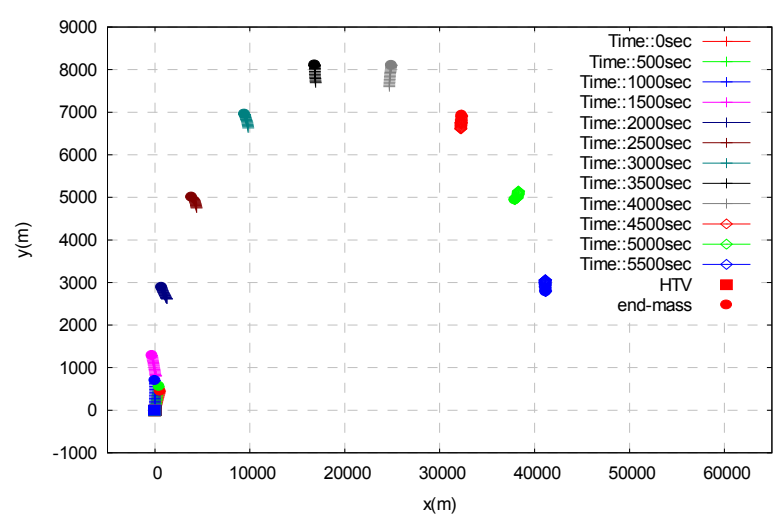

Fig. 18. Tether behavior when the tether is cut in liberating condition.

\section{Future Applications}

As is described in section 3.2, the demonstration of the ability to remove debris using a small satellite is studied for the next step toward realization of debris removal. With this concept, the DRS attaches one end of the tether to the debris object and the tether is deployed from a reel onboard the DRS with its thrusters. The DRS functions as the end-mass of the tether and de-orbits with the debris. The tether dynamics that are demonstrated by the KITE mission can facilitate the realization of many interesting operations by DRS in the future. In the following subsections, some of the DRS-based numerical simulations results are summarized.

\subsection{Tether libration suppression and CAM}

The tether libration amplitude is important for the DRS because it affects the Lorentz force for lowering the altitude: if the libration amplitude is minimized, it can efficiently de-orbit debris, without the risk of unexpected tumbling. The tether libration can be either managed by current control or suppressed by thrust applications.

The DRS' collision avoidance maneuver with the deployed tether may be possible during the de-orbiting phase. At the same time, the altitude can be lowered and tether libration can be decreased if the thrust is applied at the optimal time (Fig. 19). In this figure, the mass of the debris is assumed to be 3.4 tons and the mass of the DRS is $350 \mathrm{~kg}$. The length of the tether is
$10 \mathrm{~km}$ and the initial libration amplitude is $50^{\circ}$. It shows that the libration amplitude becomes about $5^{\circ}$, and the semi major axis of the orbit of the DRS and the debris is lowered by about $3 \mathrm{~km}$ by $\mathrm{dV} 1.5 \mathrm{~m} / \mathrm{s}$. Collision avoidance can also be achieved by switching the electric current on and off by shifting the orbital phase of the DRS and the debris object with which the collision is predicted.

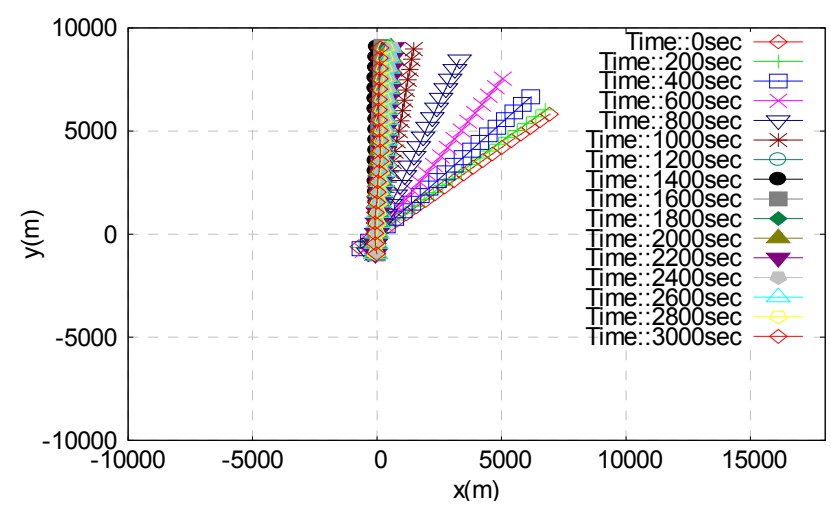

Fig. 19. Libration suppression by $\mathrm{dV} 1.5 \mathrm{~m} / \mathrm{s}$ of the DRS, using thrusters with the total thrust of $9.2 \mathrm{~N}$ for about $600 \mathrm{~s}$ starting about time $=800 \mathrm{~s}$.

\subsection{Controlled reentry}

EDT thrust cannot conduct controlled reentry because its thrust is too small. Some large debris objects may require controlled reentry to prevent the risk of casualties on the ground. After the DRS reaches a low enough altitude by EDT thrust, a controlled reentry may be conducted. The option of applying a tethered tug (i.e., a satellite that pulls the debris by a tether along the direction of the orbital velocity) has also been studied. A tether stabilized in the vertical direction can be intentionally rotated by a small thrust followed by a large thrust, which sets the necessary conditions for controlled reentry. The dynamics of the horizontal direction of the tether are difficult to predict because no restoring force will work to contrast the vertically stabilized tether. The dynamics of the tether are insignificant after they are set in the reentry trajectory (Fig. 20).

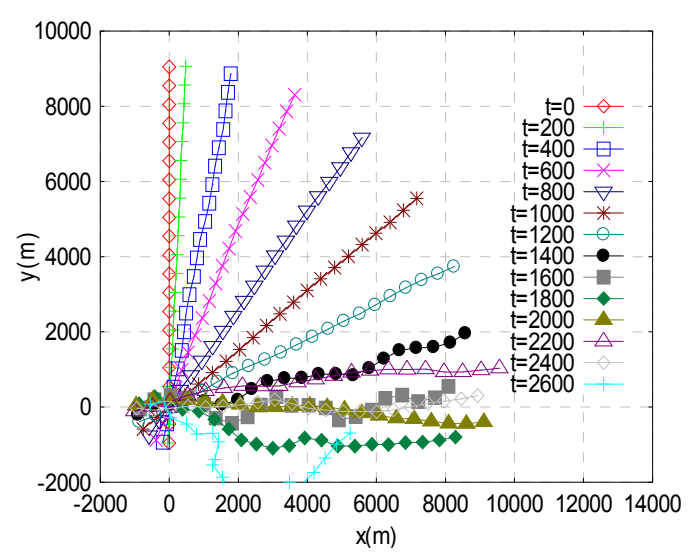

Fig. 20. Controlled reentry after an EDT operation. A small thrust $(\mathrm{dv}=$ $3.0 \mathrm{~m} / \mathrm{s}$ ) is applied at time $=0 \mathrm{~s}$ to rotate the tether, and then, large thrust burn $(\mathrm{dV}=40 \mathrm{~m} / \mathrm{s})$ is applied when the tether is almost horizontal (at time $=$ about $1500 \mathrm{~s})$. 


\section{Conclusion}

This paper provided an overview of the dynamics of the KITE tether that can be expected, which is to be launched on the HTV6. Precise numerical simulations were performed to provide information on key aspects of the mission analysis, by modeling the tether as point masses connected by springs and viscous dampers. The characteristics of EDT obtained by conducting the KITE mission will be useful not only for designing debris removal satellites in the future but also for realizing various interesting operations such as collision avoidance maneuvers and controlled reentry with a tether.

\section{References}

1) Inter-Agency Space Debris Coordination Committee (IADC) Working Group 2,: Stability of the Future LEO Environment http://www.iadc-online.org/Documents/IADC-201208,\%20Rev\%201,\%20Stability\%20of\%20Future\%20LEO\%20Envi ronment.pdf

2) Liou, J.-C.: An active debris removal parametric study for LEO environment remediation, Advances in Space Research, 47, Issue 11, 1 June (2011), pp.1865-1876.

3) Kawamoto, S., Ohkawa, Y., et al.: Strategy for Active Debris Removal Using Electrodynamic Tether, Trans. JSASS Aerospace Tech. Japan, 7, ists26 (2009), pp. Pr_2_7-12.

4) Sanmartin, J. R., Martinez-Sanchez, M. and Ahedo, E.: Bare Wire
Anodes for Electrodynamic Tethers, J. Prop. and Power, 9 38(1993), pp. 353-360.

5) Kawamoto, S., Ohkawa, Y., et al.: ACTIVE DEBRIS REMOVAL BY A SMALL SATELLITE, IAC-12- A6.7.8, 2012.

6) Aoyama, J., Kawamoto, S., et al.: Mounting Technology of the Propulsion System for De-orbiting Debris, Proceedings of the 6th Space Debris Workshop, JAXA https://repository.exst.jaxa.jp/dspace/bitstream/ais/546814/1/AA1530025014.pdf, 2014.

7) Kubota, N., Enomoto, M., et al.: Conceptual Study of Mechanical and Sensing System for Debris Capturing for PAF, 2015-r-33, 30 ISTS, 2015.

8) Mataki, T., Akahoshi, Y., et al.: Evaluation of Harpoon Tips for Debris Capture, 2015-r-29, 30 ${ }^{\text {th }}$ ISTS, 2015.

9) Ohkawa, Y., Kawamoto, S., et al.: Preparation for On-Orbit Demonstration of Electrodynamic Tether on HTV, 2015-b/IEPC301, 34 ${ }^{\text {th }}$ IEPC, 2015.

10) Tsujita, D., Kasai, T., et al.: Operational feasibility evaluation for on-orbit EDT experiments on HTV, 2015-g-03, 30 th ISTS, 2015.

11) Otsuka, A., Tsujita, D., et al.: Designing the International Framework for Active Debris Removal Operation, 2015-t-05, 30 ${ }^{\text {th }}$ ISTS, 2015.

12) Kawamoto, S., Makida, T., et al.: Precise Numerical Simulations of Electrodynamic Tethers for an Active Debris Removal System, Acta Astronautica, 59 (2006) pp.139-148.

13) Iki, K., Kawamoto, S. and Morino, Y.: Numerical Simulations of an Electrodynamic Tether Deployment from a Spool-Type Reel Using Thrusters, IAA-AAS-DyCoSS1-10-01, AAS 12-368, 2010.

14) Iki, K., Kawamoto, S. et. al.: Expected On-orbit Tether Deployment Dynamics on the KITE Mission, ISTS-r-27, 30 ${ }^{\text {th }}$ ISTS, 2015. 